\title{
Adaptive Super-Candidate Based Approach for Detection and Classification of Drusen on Retinal Fundus Images
}

\author{
Vaanathi Sundaresan ${ }^{1}$, Keerthi Ram ${ }^{2}$, Kulasekaran Selvaraj ${ }^{2}$, Niranjan Joshi ${ }^{2}$ \\ and Mohanasankar Sivaprakasam ${ }^{1,2}$ \\ 1 Indian Institute of Technology Madras (IITM), India \\ ${ }^{2}$ Healthcare Technology Innovation Centre, IITM, India
}

\begin{abstract}
Identification and characterization of drusen is essential for the severity assessment of age-related macular degeneration (AMD). Presented here is a novel super-candidate based approach, combined with robust preprocessing and adaptive thresholding for detection of drusen, resulting in accurate segmentation with the mean lesion-level overlap of 0.75 , even in cases with non-uniform illumination, poor contrast and confounding anatomical structures. We also present a feature based lesionlevel discrimination analysis between hard and soft drusen. Our method gives sensitivity of $80 \%$ for high specificity above $90 \%$ and high sensitivity of $95 \%$ for specificity of $70 \%$ on representative pathological databases (STARE and ARIA) for both detection and discrimination.
\end{abstract}

\section{Introduction}

Age-related macular degeneration (AMD) is one of the leading causes of blindness among aged population. Various stages of AMD are characterized in retinal color fundus images by the presence of drusen, which are yellowish blobs with varying intensity, color and edge strength (shown in Fig. 1).
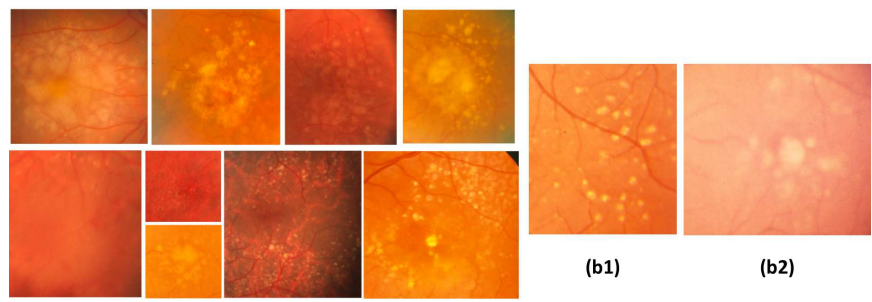

(a)

Fig. 1. (a) Drusen of various size and types shown. (b1) Hard drusen, (b2) Soft drusen

Early AMD is identified by hard drusen (HD), which appear as localized, brighter structures with relatively sharper edges on retinal images. As the disease progresses into intermediate stage, several hard drusen spread and coalesce into very dull, well-spread soft drusen (SD). Reliable detection of drusen and their discrimination into HD and SD in retinal images form the core of a computerassisted AMD grading system.

X. Chen, M. K. Garvin, J. Liu, E. Trucco, Y. Xu (Eds.): OMIA 2015, Held in Conjunction with MICCAI 2015, Munich, Germany, Iowa Research Online, pp. 81-88, 2015. Available from: http://ir.uiowa.edu/omia/2015_Proceedings/2015/ 


\subsection{Prior work}

Existing work for drusen detection in retinal fundus images includes multi-level thresholding [1], pixel-wise clustering [2], template matching [3], classification based on intensity, texture and contrast based features [4] and adaptive thresholding (HALT) [5]. Compared to non-specific signs like exudates, drusen appear duller as they occur in sub-retinal layers. Moreover, automatic drusen detection is hindered by normal anatomical structures such as retinal nerve fiber layer (RNFL), optic disc and choroidal vessels in tessellated retina and adverse imaging conditions such as non-uniform illumination and low contrast. Therefore various preprocessing techniques such as iterative background modeling [1], [6], multi-level histogram equalization [5] has been developed in prior work. While identification of hard drusen is well attempted and utilized directly in the AMD grading systems, reported literature on the detection of soft drusen are scarce.

$\mathrm{HD}$ are generally smaller $(<63 \mu \mathrm{m})$ than $\mathrm{SD}(63 \mu \mathrm{m}$ to $\approx 1 \mathrm{~mm})$. Utilizing this difference in size, classification of drusen has been done in prior work based on size [7], [2] and area [1], [6]. However, reliability of size and area measurement is limited by factors such as angle of acquisition (magnification), inaccurate segmentation and clumped drusen being misclassified as isolated drusen. Apart from drusen size, discrimination between hard and soft drusen using texture based AM-FM features has been done on localized $40 \times 40$ patches [8].

In our work, we suppress normal anatomical structures such as choroidal vessels due to tessellated retina and RNFL by a robust preprocessing technique. We present a novel adaptive thresholding technique for reliable identification of initial foreground regions, which are refined by a super-candidate based approach. We utilize a supervised method for discriminating between HD and SD based on their structural characteristics, apart from size.

\section{Method}

The complete flow of our proposed method for drusen detection and discrimination is shown in Fig. 2. Our method consists of following steps: 1. Localization of macular region and preprocessing, 2. Initial foreground extraction, 3. Supercandidate formation, 4 . Supervised ranking of super-candidates to select drusen, 5. Supervised discrimination between hard and soft drusen.

The input to preprocessing uses color opponent channels which were observed to provide better visibility of drusen and suppress choroidal vessels. The difference of blue-yellow $I_{b y}$ and red-green color $I_{r g}$ opponent channels is used as the input channel $I_{\text {in }}$ (Fig. 3 (a)) for subsequent steps.

$$
I_{i n}=I_{b y}-I_{r g}, \quad \text { where } I_{b y}=\left(I_{r} / 2+I_{g}-2 I_{b}\right) / \sqrt{6} ; I_{r g}=\left(I_{r} / 2-I_{g}\right) / \sqrt{2}
$$

The preprocessing step includes non-linear contrast enhancement, gamma correction (for selective saturation of drusen) and histogram equalization. We use background adaptive thresholding method to obtain initial foreground regions $F_{i}$. Within each region of $F_{i}$, with respect to local contrast, drusen appear as 


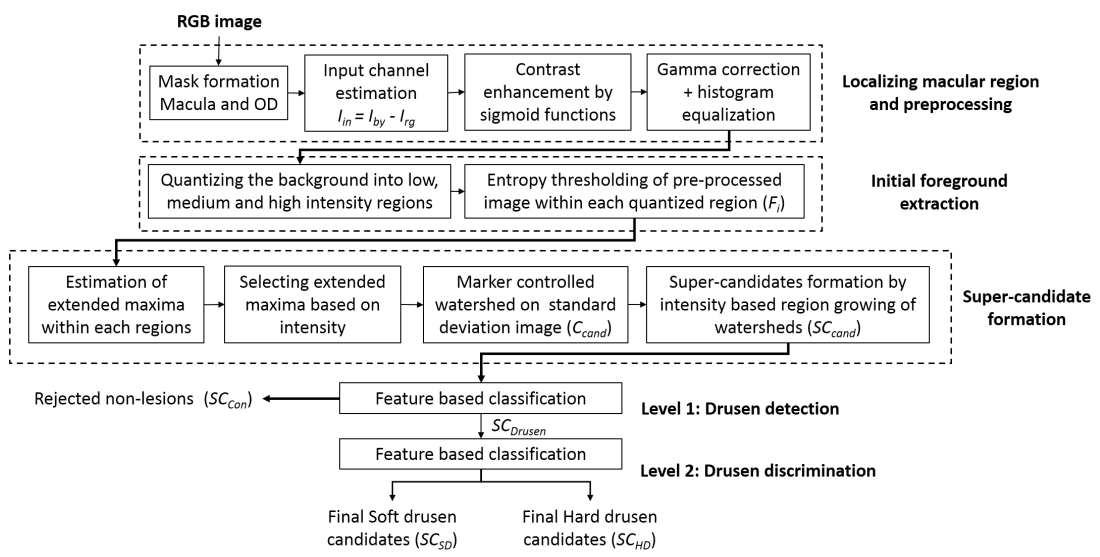

Fig. 2. Flowchart depicting the process of drusen detection and discrimination

relatively bright regions. Hence we obtain local maxima within $F_{i}$ and form candidates around individual local maxima. In order to segment larger faint soft drusen without fragmenting, we emulate the coalescence of drusen by grouping candidates with similar intensity characteristics to form super-candidates. We rank super-candidates by a supervised classifier to select drusen. We further discriminate drusen into HD and SD based on structurally descriptive features.

\subsection{Localization of macular region and preprocessing}

On the input channel $I_{i n}$, we additively apply series of sigmoid functions as transfer function (Fig. 3 (1)) on the intensities. Zero-crossing points of the sig-

(1)

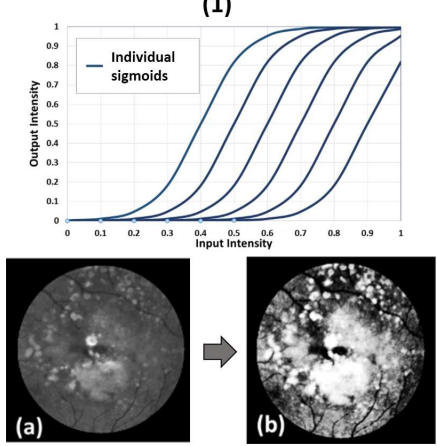

(2)

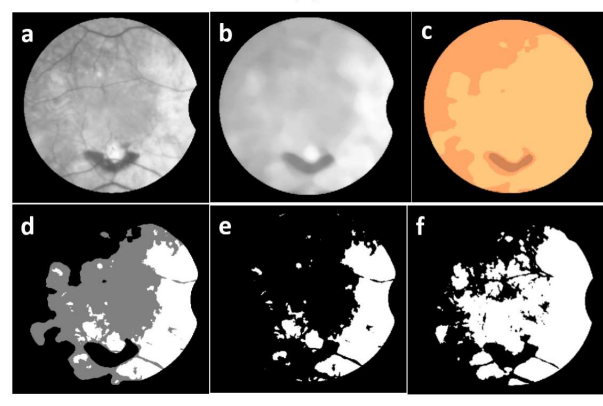

Fig. 3. (1) The individual sigmoid functions shown in blue, (1a) Input channel $I_{i n}$, (1b) Preprocessed channel $I_{\text {pre }}$, (2a) Input channel $I_{i n},(2 \mathrm{~b})$ Estimated background, (2c) Indexed image based on 3 intensity levels of background, (2d) Entropy threshold within a region corresponding to single intensity level, (2e) Initial foreground regions $F_{i},(2 \mathrm{f})$ Result of entropy threshold without background indexing (for comparison only)

moid functions are shifted to selectively enhance the intensities which are above 
background level. This transfer function enhances lesions of different intensities towards same brighter level. This step is followed by histogram equalization to get $I_{\text {pre }}$ (Fig. $3(1 \mathrm{~b})$ ). We detect the optic disc (OD) and macula by the method described in [9], and consider a circular region of interest (ROI), with fovea as center and 1.5 times disc diameters as radius, and mask the OD if it falls within the ROI.

\subsection{Initial foreground extraction}

We estimate the background of $I_{\text {in }}$ (Fig. $3(2 \mathrm{~b})$ ) by $35 \times 35$ median filtering and convert it to an indexed image with 3 intensity levels: $l_{\text {low }}$ maps to background and dull soft drusen, $l_{\text {high }}$ maps to bright drusen and $l_{\text {int }}$ maps to medium intensity drusen. The entropy threshold on $I_{\text {in }}$ within the regions corresponding to each intensity level of background gives the less noisy initial foreground regions $\left(F_{i}\right)$ (Fig. 3(2d),(2e)).

\subsection{Super-candidates formation}

Super-candidates: Super-candidates are contiguous regions with similar intensity characteristics, formed by merging candidates, utilizing grouping cues such as adjacency and intensity. Candidates are regions with low intra-region intensity variance formed around individual local maxima. In order to segment drusen of various sizes, candidates need to be grouped into super-candidates by considering region-wise intensity homogeneity rather than pixel-wise.

Candidate formation by marker controlled watershed: We divide $F_{i}$ into candidates based on local maxima and ridges such that we get candidates around each local maximum. We use marker controlled watershed on $I_{\text {pre }}$ within each region of $F_{i}$, dividing it into individual drusen candidates. We get the basins (internal marker) from local maxima of H-maxima transform (yellow regions in

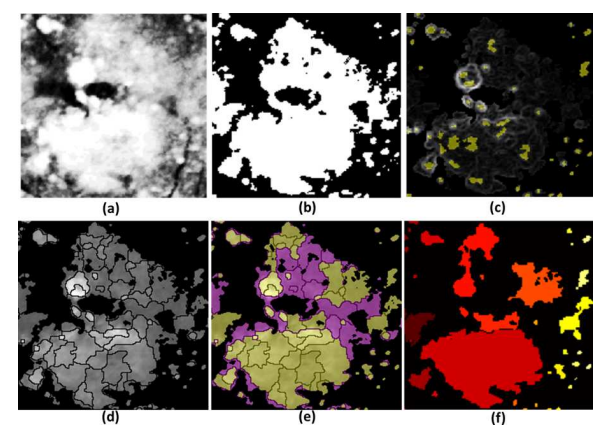

Fig. 4. Magnified section of ROI. (a) Preprocessed channel $I_{\text {pre }}$, (b) Foreground regions $F_{i}$, (c) Edges of the standard deviation filter shown along with the local maxima regions (shown in yellow), (d) Watersheds indicating drusen candidates, (e) Drusen (yellow) and background (magenta) super-candidates (color shown for illustration only) (f) Classified drusen super-candidates $S C_{\text {Drusen }}$ 
fig. 4(c)) and the ridge lines (external marker) from the edges of standard deviation filter (Fig. 4(c)) applied on $I_{\text {pre }}$. Guided by the above ridges and basins of the marker, we obtain individual candidates by watershed transform (Fig. 4(d)). Conditional merging to form super-candidates: We merge the adjacent watersheds having almost similar region-wise mean intensities (difference in their mean intensities $\leq 0.01)$ to form super-candidates $\left(S C_{\text {cand }}\right)$ (Fig. $4(\mathrm{e})$ ).

\subsection{Supervised classification of super-candidates to select drusen}

Among the detected super-candidates $\left(S C_{c a n d}\right)$, there could be non-drusen regions such as edges of RNFL and regions between drusen. Hence we consider the following features, including anatomy and vessel based features, in order to differentiate drusen super-candidates $S C_{\text {Drusen }}$ from the above confounders.

- Mean, median, mode and standard deviation values of 8 color auto-correlogram on the RGB color space within the bounding box of $S C_{\text {cand }}(i)$ within pixel radii of 1 and 3.

- Contrast, correlogram, energy and homogeneity values of gray level co-occurrence matrix (GLCM) within the bounding box of $S C_{\text {cand }}(i)$

- Mean perpendicular distances from centroid $S C_{\text {cand }}(i)$ to the disc center and to the nearest point on the parabola fitted on retinal main arcade [9]. The distances are normalized by the retinal raphe length.

- Difference between the mean intensity value within $S C_{\text {cand }}(i)$ and local background intensity within its bounding box

- Difference between the mean intensity value within $S C_{c a n d}(i)$ and global background intensity

- Ratio between maximum intensity value within $S C_{\text {cand }}(i)$ and local background intensity within its bounding box

- Mean, median and standard deviation values of intensity of $I_{\text {pre }}$ within $S C_{c a n d}(i)$

- Mean, median and standard deviation values of intensity of the red, green, blue and opponent color spaces $\left(I_{b y}\right.$ and $\left.I_{r g}\right)$ within $S C_{\text {cand }}(i)$

- Mean value of entropy of green, opponent color spaces ( $I_{b y}$ and $\left.I_{r g}\right)$ and $I_{p r e}$ within $S C_{\text {cand }}(i)$ excluding the vessel pixels.

- Mean gradient values of green, opponent color spaces $\left(I_{b y}\right.$ and $\left.I_{r g}\right)$ and $I_{p r e}$ within $S C_{\text {cand }}(i)$

We use random forest classifier $R F_{1}$, a two-class ensemble classifier, to assign a confidence score between 0 and 1 , based on the posterior probability of the features. We select the super-candidates $S C_{\text {cand }}$ with higher score as $S C_{\text {Drusen }}$ (Fig. 4(f)) by thresholding their confidence scores.

\subsection{Supervised discrimination between hard and soft drusen}

Hard and soft drusen are different from each other in color, texture, spread, edge strength, symmetry and their spatial proximity, apart from size. HD are brighter, more localized with distinct edges and circular blobs compared to SD. We exploit this difference in their characteristics and extract following features for each drusen super-candidate $S C_{\text {Drusen }}(i)$ for discriminating $\operatorname{HD}\left(S C_{H D}\right)$ from $\operatorname{SD}\left(S C_{S D}\right)$. 
- Difference between area enclosed by different level of contours, in 10 level isocontour analysis within $S C_{\text {Drusen }}(i)$.

- Mean and standard deviation values of the phase congruency [10] values of $I_{\text {pre }}$ within $S C_{\text {Drusen }}(i)$ and along its edge

- Mean values of the first and second order steerable filter response of $I_{\text {pre }}$ at 18 different orientations, within $S C_{\text {Drusen }}(i)$ and along its edge

- Mean of eigenvalues $\lambda_{1}, \lambda_{2}$ and their ratio $\left(\lambda_{1} / \lambda_{2}\right)$ of the Hessian matrix on $I_{p r e}$ along the edge of $S C_{\text {Drusen }}(i)$

- Mean and standard deviation values of phase symmetry [10] on $I_{\text {pre }}$ within $S C_{\text {Drusen }}(i)$

- Mean value of the fast radial transform of $I_{\text {pre }}$ within $S C_{\text {Drusen }}(i)$

- Mean and standard deviation values of the CMYK, luv and opponent color spaces ( $I_{b y}$ and $I_{r g}$ ) within $S C_{\text {Drusen }}(i)$

- Maximum and mean values of the 13 rotationally invariant filters of Schmid filter bank response of $I_{\text {pre }}$ within $S C_{\text {Drusen }}(i)$

- Maximum and standard deviation values of Laplacian of Gaussian (LoG) filter at 4 scales within $S C_{\text {Drusen }}(i)$

We use random forest classifier $R F_{2}$ for the second level of classification in order to discriminate HD and SD.

\section{Results and discussion}

Database and ground truth: We evaluated the proposed method on STARE and ARIA databases consisting of 214 challenging images (113 - images with drusen and 101 normal) with wide variation in contrast and illumination. The ground truth has been marked for HD and SD at lesion-level by a retinal specialist. The training set $(T r)$ consists randomly selected 107 images with 3170 drusen regions (2850 HD and $320 \mathrm{SD}$ ), while false alarms in the detection are used as confounders for training the classifier.

Training specifications for classifier: We obtained 52 features at the detection stage and 203 features at discrimination stage. We trained $R F_{1}$ with 750 trees and 8 randomly selected features for $\operatorname{Tr}$ consisting of 3170 drusen regions and 4256 confounders. Similarly, we trained $R F_{2}$ with 1000 trees and 15 randomly selected features for $\operatorname{Tr}$ consisting of $2850 \mathrm{HD}$ and $320 \mathrm{SD}$ regions.

Performance evaluation: Performance of $R F_{1}$ was evaluated on a test set (Ts) of 107 images consisting of 3313 drusen and 4073 confounders. We consider a super-candidate as true positive if Szymkiewicz-Simpson overlap coefficient (overlap) between detected drusen $S C_{\text {Drusen }}$ and the ground truth $G T$, given by overlap $\left(S C_{\text {Drusen }}, G T\right)=\left|S C_{\text {Drusen }} \cap G T\right| / \min \left(\left|S C_{\text {Drusen }}\right|,|G T|\right)$, is more than 0.6. Experimentally, we observed the better performance for overlap range of 0.45 - 0.6. Fig. 5(a) shows the mean lesion-level overlap value of segmented drusen regions with respect to ground truth, for the test images $T s$ plotted for different confidence score threshold values. As shown in Fig. 5(a), super-candidates gives better segmentation (overlap - 0.75) than the candidate stage (overlap - 0.13) at the optimal threshold value of 0.4 due to our selective region merging. Performance of $R F_{2}$ was evaluated on $T s$ consisting of $3119 \mathrm{HD}$ and $294 \mathrm{SD}$. ROC curves for drusen detection and discrimination on $T s$ are shown in fig. 5(b). The optimal performance points occur at 


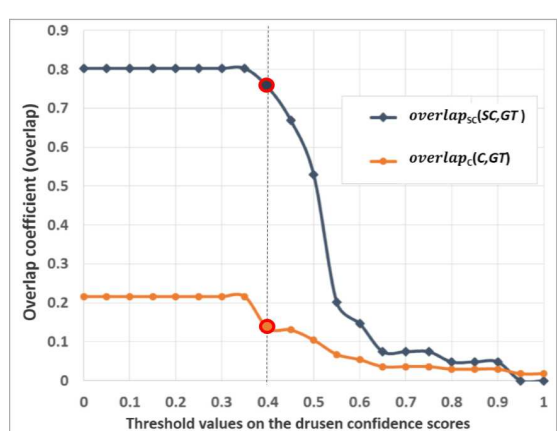

(a)

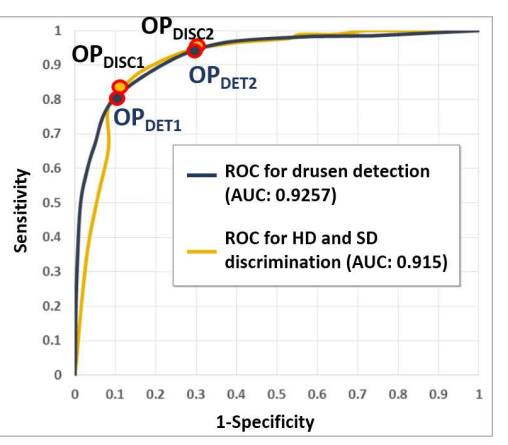

(b)

Fig. 5. (a) Values of overlap shown at different levels of confidence score thresholds for super-candidate stage $S C$ and candidate stage $C$ (blue and orange curves respectively) of drusen detection. The highlighted dots show the mean lesion-level overlap at operating point threshold of 0.4 , (b) ROC curves for drusen detection and discrimination (blue and yellow curves respectively). Operating points $O P_{D E T_{1}}, O P_{D E T_{2}}$ and $O P_{D I S C_{1}}, O P_{D I S C_{2}}$ corresponds to drusen detection and discrimination respectively.

Table 1. Comparison of our method with existing methods

\begin{tabular}{|c|c|c|c|}
\hline Methods & Analysis & Database, No of images & Performance \\
\hline Rapantikos et al [5] & drusen detection &,- 23 & $\begin{array}{c}\text { Sens, Spec }->96 \% \text { except } 1 \\
\text { image }(88 \%)\end{array}$ \\
\hline Brandon et al [2] & drusen detection & STARE, 113 & Sens - $86 \%, \underset{87 \%}{\text { Spec }}-93 \%$, Acc - \\
\hline Mora et al [6] & $\begin{array}{l}\text { discrimination (size) } \\
\text { drusen detection } \\
\text { discrimination (area) }\end{array}$ & $\begin{array}{l}\text { STARE, } 113 \\
-, 22 \\
-.22\end{array}$ & $\begin{array}{c}\text { Acc- }-71 \% \\
\text { Sens - } 74 \%, \text { Spec - } 94 \% \\
\text { Sens - } 63 \%, \text { Spec }-96 \%\end{array}$ \\
\hline $\begin{array}{l}\text { Smit } \\
\text { Wong }\end{array}$ & $\begin{array}{l}\text { (area) } \\
\text { ction }\end{array}$ & $\begin{array}{c}-, 358 \\
\text { THALIA, } 350\end{array}$ & $\begin{array}{l}\text { Sens }-87.3 \%, \text { Spec }-95.3 \% \\
\text { Precision - } 95.46 \pm 0.94\end{array}$ \\
\hline Zheng et al [12] & $\begin{array}{c}\text { discrimination (area) } \\
\text { Small } \\
\text { Medium } \\
\text { Large } \\
\text { drusen detection }\end{array}$ & CAPT & $\begin{array}{c}\text { Acc } \\
84.06-92.64 \% \\
89.89-96.06 \% \\
90.67-95.16 \% \\
\text { Sens - } 82 \%, \text { Spec }-75 \%, \text { Acc - }\end{array}$ \\
\hline Barriga et al [8] & $\mathrm{HD} / \mathrm{SD}$ discrimi &,- 5 & $\begin{array}{c}80 \% \\
\text { Acc }-96 \% \text { for } 120 \text { patches ( } 5 \\
\text { images) }\end{array}$ \\
\hline \multirow[t]{2}{*}{ Proposed method } & drusen detection & STARE, ARIA, 214 & $\begin{array}{l}\text { Sens }-80 \% \text {, Spec - } 90 \% \text {; Sens - } \\
95 \% \text {, Spec }-70 \%, \text { AUC }-0.9257\end{array}$ \\
\hline & $\mathrm{HD} / \mathrm{SD}$ discrimination & STARE, ARIA, 214 & $\begin{array}{l}\text { Sens }-80 \% \text {, Spec }-92 \% \text {; Sens - } \\
95 \% \text {, Spec }-70 \% \text {, AUC }-0.915\end{array}$ \\
\hline
\end{tabular}

$O P_{D E T_{1}}\left(\right.$ sens $-80 \%$, spec-90\%), $O P_{D E T_{2}}($ sens $-95 \%$, spec-70\%) for drusen detection at the confidence score threshold value of $0.4,0.25$ and $O P_{D I S C_{1}}$ (sens$80 \%$, spec $-92 \%), O P_{D I S C_{1}}($ sens $-95 \%$, spec $-70 \%)$ for HD and SD discrim-
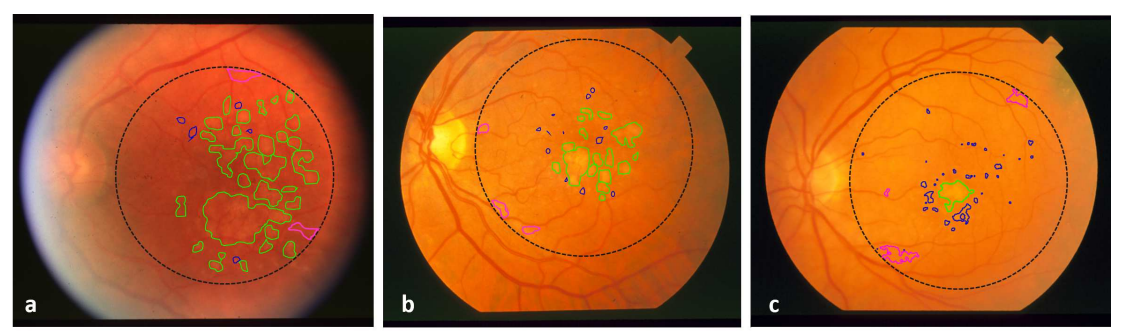

Fig. 6. Final output shown on the retinal images. Magenta color - candidates rejected in the detection stage; Blue - hard drusen and green - soft drusen 
ination at the threshold value of $0.5,0.3$. Super-candidate gives reliable segmentation and anatomy based features reduces false positives such as RNFL as shown in final results in Fig. 6. As shown in Table 1, our method gives good performance for drusen detection and discrimination.

\section{Conclusion}

We present a robust method for the detection of drusen with $80 \%$ sensitivity at $90 \%$ specificity and $95 \%$ sensitivity at $70 \%$ specificity, which is comparable with existing work. Our method uses a novel preprocessing technique which reduces the effect of background intensity variations on drusen detection. The main contributions of our approach are super-candidate based segmentation and a set of characteristic features for discrimination of drusen. The discrimination of hard and soft drusen yields $80 \%$ sensitivity at $92 \%$ specificity and $95 \%$ sensitivity at $70 \%$ specificity, which is better than drusen discrimination based on size/area. In our future work, we aim to detect drusen in presence of other bright pathological signs, and quantify the extent and severity of disease signs which would aid in screening/grading of AMD in color retinal fundus images.

\section{References}

1. Smith, R.T., et al.: Drusen analysis in a human-machine synergistic framework. Archives of ophthalmology 129(1) (2011) 40-47

2. Brandon, L., Hoover, A.: Drusen detection in a retinal image using multi-level analysis. In: MICCAI. Springer (2003) 618-625

3. Freund, D.E., et al.: Automated detection of drusen in the macula. In: ISBI, IEEE (2009) 61-64

4. Niemeijer, M., et al.: Automated detection and differentiation of drusen, exudates, and cotton-wool spots in digital color fundus photographs for diabetic retinopathy diagnosis. IOVS 48(5) (2007) 2260-2267

5. Rapantzikos, K., et al.: Detection and segmentation of drusen deposits on human retina: Potential in the diagnosis of age-related macular degeneration. Medical image analysis $\mathbf{7}(1)$ (2003) 95-108

6. Mora, A.D., et al.: Automated drusen detection in retinal images using analytical modelling algorithms. Biomedical engineering online 10(1) (2011) 59

7. Köse, C., et al.: A statistical segmentation method for measuring age-related macular degeneration in retinal fundus images. J. med sys 34(1) (2010) 1-13

8. Barriga, E.S., et al.: Multi-scale am-fm for lesion phenotyping on age-related macular degeneration. In: CBMS, IEEE (2009) 1-5

9. Sundaresan, V., et al.: Integrated approach for accurate localization of optic disc and macula. In: Proc of the OMIA, MICCAI (2014) 97-104

10. Kovesi, P.: Invariant measures of image features from phase information. PhD thesis, University of Western Australia (1996)

11. Wong, D.W.K., et al.: Thalia-an automatic hierarchical analysis system to detect drusen lesion images for amd assessment. In: ISBI, IEEE (2013) 884-887

12. Zheng, Y., et al.: An automated drusen detection system for classifying age-related macular degeneration with color fundus photographs. In: ISBI, IEEE (2013) 14481451 